\title{
Effect of Helicobacter pylori Eradication on Migraine Headache: A Before -After Clinical Trial
}

\begin{abstract}
Keywords: Migraine disorders; Helicobacter pylori; Headache; Clinic al trial

Abstract

Background: Migraine is the most frequent type of primary headache. It has been demonstrated that $\mathrm{H}$. pylori infection eradication has a possible role in the reduction of migra ine headache but needs to be examined in different settings using Urea Breath Test (UBT). This clinic al trial was conducted to evaluate the effect of the $\mathrm{H}$. pylori eradication on improvement of migraine.

Methods: This c linic al trial was conducted in Farshchian Hospital in 2012. Eighty patients with migraine headache who were seropositive for $\mathrm{H}$. pylori infection were enrolled in the trial. Migraine was defined according to the Intemational Headache Society criteria. H. pylori infection was diagnosed according to the detection of anti-H. pylor Ig a ntibody in the blood sample. Standard four-drug protocol for $\mathrm{H}$. pylori eradication was prescribed with Omeprazole $20 \mathrm{mg}$ Bid, Bismuth $240 \mathrm{mg}$ Bid, Tetracycline $500 \mathrm{mg}$ Qid, and Metronidazole $500 \mathrm{mg}$ Bid for four weeks. The outcomes of interest were the severity and frequency of migraine headache.
\end{abstract}

Results: The mean scores of the severity of headache decreased from 8.20 before $H$. pylori eradication to 2.91 after that $(P \varangle 0.001)$ and the frequency of headache from 7.45 to 2.73 ( $P \varangle 0.001$ ). The reduction in severity and frequency of headache was much more in patients with negative UBT $(\mathrm{P}<0.001)$ compared to those with positive test results $(\mathrm{P}=0.002)$.

Conclusion: This trial indicated that $\mathrm{H}$. pylori eradication can efficiently reduce the severity and frequency of migraine headache shortly after treatment in patients who were infected with $\mathrm{H}$. pylori. However, the long-tem effect of the treatment was not explored and needs to be investigated.

\section{Introduction}

Migraine is the most frequent kind of primary headache affecting about $18 \%$ of females and $6 \%$ of males in the general population [1] It has been demonstrated that Helicobacter pylori (H. pylori) infection has a possible role in the precipitation of migraine while other data support a single co-occurrence of $H$. pylori and migraine [2,3].

Association between $H$. pylori infection and various extraintestinal pathologies, such as migraine, has recently been addressed in some literatures [4-10]. Some studies have shown that the inflammatory response caused by $H$. pylori infection may affect migraine through an immune-mediated release of cytokines and other substances endowed with vasospastic and procoagulant properties [11-14]. However, more evidence is needed to confirm the relationship between $H$. pylori infection and migraine in different settings. Furthermore, evaluation of the patients in term of urea breath test (UBT) results is another issue that was not addressed in the previous literatures. Accordingly, this clinical trial was conducted to evaluate the effect of the H. pylori

\section{Journal of Neurology and Psychology}

Shahir Mazaheri ${ }^{1}$, Amir Majlesi $^{2 *}$, Jalal Poorolajal ${ }^{3}$, NiloofarHemati ${ }^{2}$ and Alireza Khalilian ${ }^{2}$

${ }^{1}$ Department of Neurology, School of Medicine, Hamadan University of Medical Sciences, Hamadan, Iran

${ }^{2}$ Department of Internal Medicine, School of Medicine, Hamadan University of Medical Sciences, Hamadan, Iran

${ }^{3}$ Modeling of Non-communicable Diseases Research Center, Department of Epidemiology \& Biostatistics, School of Public Health, Hamadan University of Medical Sciences, Hamadan, Iran

*Address for Correspondence

Amir Majlesi, MD, Department of Internal Medicine, School of Medicine, Hamadan University of Medical Sciences, Hamadan, Iran 6517838695, Tel: 0098811 2023; Fax: 0098811 2023; E-mail: majlesi200@yahoo.com

Copyright: (c) 2014 Mazaheri S, et al. This is an open access article distributed under the Creative Commons Attribution License, which permits unrestricted use distribution, and reproduction in any medium, provided the original work is properly cited.

Submission: 01 July 2014

Accepted: 01 August 2014

Published: 06 August 2014

Reviewed \& Approved by: Dr. Pradeep Sahota, Professor, Department of Neurology, University of Missouri School of Medicine, USA

eradication in a population of patients affected by migraine and to verify whether the eradication of the bacterium may represent a novel, encouraging approach to the management of patients with migraine.

\section{Materials and Methods}

This before-after clinical trial was conducted in the neurologic clinic of Farshchian Hospital, affiliated with Hamadan University of Medical Sciences, from January to December 2012. Written informed consent was received from all patients. The Ethic Committee of the Hamadan University of Medical Sciences approved the consent procedure and the trial (D-P-9-35-16-3586). The protocol was registered by the Iranian Registry of Clinical Trials (IRCT201202018896N1) and is available at:

http://www.irct.ir/searchresult.php?keyword=Helicobacter\&id= 8896\&number $=1 \&$ field $=$ c \&prt $=22 \&$ total $=10 \& \mathrm{~m}=1$.

Eighty patients with migraine headache who were seropositive for $H$. pylori infection were enrolled in this trial. Migraine was defined according to the International Headache Society (IHS) criteria. Patients with a pulsating headache with nausea, vomiting, photophobia, phonophobia, and seropositive $H$. pylori patients were included. The patients with the following criteria were excluded from the study: (a) already received $H$. pylori intervention treatment; (b) hypertension; (c) fever due to any causes; (d) received acute medical treatment of migraine; or (e) having brain tumor. All patients received migraine prophylaxis including imipramine (10 $\mathrm{mg}$ daily) and sodium valproate (200 $\mathrm{mg}$ daily) and were recommended to continue regular lifestyle during the study.

H. pylori infection was diagnosed according to the detection of anti-H. pylori IgG antibody in the blood sample taken from the patients. Blood samples were labeled immediately after taking blood sera stored at $2-8{ }^{\circ} \mathrm{C}$ for 5 days. IgG antibodies to H. pylori 
Citation: Mazaheri S, Majlesi A, Poorolajal J, Hemati N, Khalilian A. Effect of Helicobacter pylori Eradication on Migraine Headache: A Before -After Clinical Trial. J Neurol Psychol. 2014;2(2): 4.

were detected using a rapid Enzyme Linked Immune Sorbent Assay (ELISA). The procedure was performed according to the manufacturer's instruction and recommended measures were chosen for diagnosing IgG of $H$. pylori. Samples with IgG values less than 15 $\mathrm{UR} / \mathrm{ml}$ were considered nonreactive for anti- $H$. pylori IgG antibodies. Values between 15 to $30 \mathrm{UR} / \mathrm{ml}$ were considered questionable. Values higher than $30 \mathrm{UR} / \mathrm{ml}$ were considered reactive. The sensitivity and specificity of the method was $95.8 \%$ and $96.2 \%$ respectively based on the manufacture instruction (For more information see http://www. radim.it).

The outcomes of interest were the severity and frequency of migraine headache. The outcomes were evaluated before and four weeks after $H$. pylori eradication using a well-structured questionnaire. The severity of the headache was measured using the Visual Analog Scale (VAS), on a scale of 0 to 10 . Zero denoted no pain, and 10 denoted the most severe pain that the patient had ever experienced. The frequency of headache was assessed with the number of headache attacks per month.

Standard four-drug protocol for $H$. pylori eradication was performed with Omeprazole $20 \mathrm{mg}$ Bid, Bismuth $240 \mathrm{mg}$ Bid, Tetracycline $500 \mathrm{mg}$ Qid, Metronidazole $500 \mathrm{mg}$ Bid for four weeks. The patients were examined for complete eradication after intervention using Urea Breath Test (UBT). The values less than 25 were considered negative. The values from 25 to 50 were considered borderline. The values higher than 50 were considered positive. According to the manufacturer's instruction, the both sensitivity and specificity of the UBT were 95\% (For more information see http:// www.kibion.com).

The paired $t$-test was used for comparing the severity and frequency of migraine headache before and after intervention. All statistical analyses were performed at 0.05 significance level using statistical software Stata 12 (StataCorp, College Station, TX, USA).

\section{Results}

We identified 118 patients, 34 patients were excluded (24 not meeting inclusion criteria, 4 declined to participate, and 2 due to other reasons) and 84 were eligible and enrolled into the study. Two patients lost to follow-up and two refused to follow-up. Eventually, 80 patients remained for analysis (Figure 1). Sixty-two patients were women and 18 were men. The mean age of the patients was 29.91 years $(\mathrm{SD}=7.99)$.

The mean severity and frequency of the migraine headache before and after the intervention is given in Table 1. According to the results, the mean scores of the severity of headache decreased from

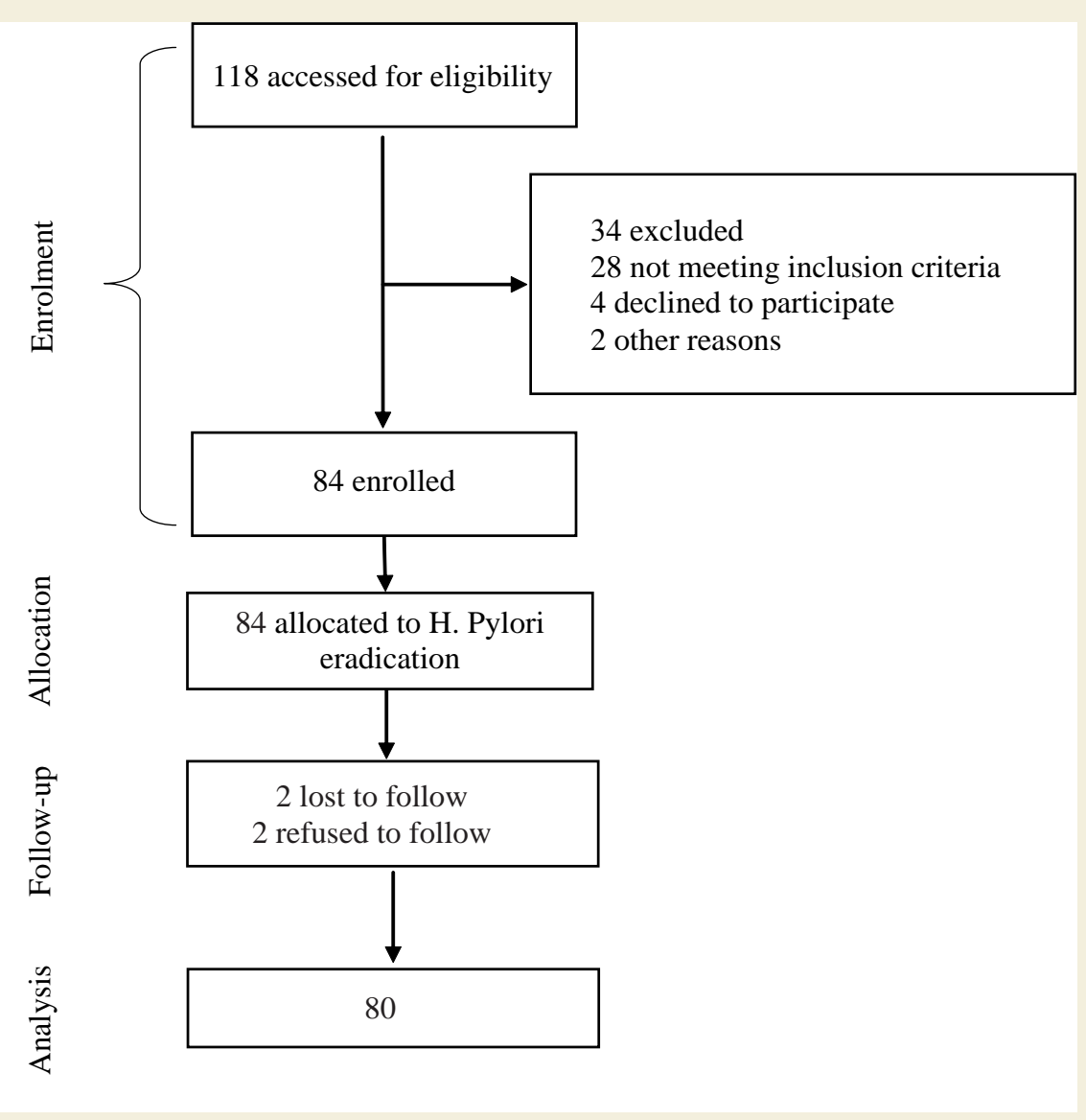

Figure 1: Flow diagram of the progress through the phases of the enrolment, follow-up, and analysis.

We identified 118 patients, 34 patients were excluded (24 not meeting inclusion criteria, 4 declined to participate, and 2 due to other reasons) and 84 were ineligible and enrolled into the study. Two patients lost to follow-up and two refused to follow-up. Eventually, 80 patients remained for analysis. 
Citation: Mazaheri S, Majlesi A, Poorolajal J, Hemati N, Khalilian A. Effect of Helicobacter pylori Eradication on Migraine Headache: A Before -After Clinical Trial. J Neurol Psychol. 2014;2(2): 4.

ISSN: 2332-3469

Table 1: Mean severity and frequency of migraine attacks before and after intervention by Urea Breath Test (UBT) results.

\begin{tabular}{|c|c|c|c|c|c|c|c|}
\hline \multirow[b]{2}{*}{ Variable } & \multicolumn{2}{|c|}{ Before intervention } & \multicolumn{2}{|c|}{ After intervention } & \multicolumn{2}{|c|}{ Difference } & \multirow[b]{2}{*}{$P$ value } \\
\hline & Mean & SD & Mean & SD & Mean & SD & \\
\hline \multicolumn{8}{|c|}{ Total (80 patients) } \\
\hline Pain severity & 8.20 & 1.48 & 2.91 & 1.64 & 5.29 & 2.08 & 0.001 \\
\hline Pain frequency & 7.45 & 1.72 & 2.73 & 1.56 & 4.73 & 2.07 & 0.001 \\
\hline \multicolumn{8}{|c|}{ UBT negative (71 patients) ${ }^{a}$} \\
\hline Pain severity & 8.30 & 1.46 & 2.56 & 1.31 & 5.73 & 1.72 & 0.001 \\
\hline Pain frequency & 7.51 & 1.71 & 2.41 & 1.26 & 5.10 & 1.85 & 0.001 \\
\hline \multicolumn{8}{|c|}{ UBT positive (9 patients) ${ }^{a}$} \\
\hline Pain severity & 7.44 & 1.51 & 5.67 & 1.41 & 1.78 & 1.20 & 0.002 \\
\hline Pain frequency & 7.00 & 1.80 & 5.22 & 1.48 & 1.78 & 1.20 & 0.002 \\
\hline
\end{tabular}

aThere was no difference between the UBT+ and UBT- patients in term of age $(P=0.155)$, gender $(P=0.094)$, and educational level $(P=0.669)$

8.20 before $H$. pylori eradication to 2.91 after that $(P<0.001)$ and the frequency of headache from 7.45 to $2.73(P<0.001)$.

The severity and frequency of migraine headache were evaluated before and after intervention among patients with negative UBT and those with positive UBT separately (Table 1). The severity and frequency of headache diminished significantly after $H$. pylori eradication in both groups. However, the reduction in severity and frequency of headache was much more in patients with negative UMT compared to those with positive UBT.

\section{Discussion}

According to our findings, $H$. pylori eradication was associated with a significant decrease in both severity and frequency of migraine attacks. Because of small sample size, we could not assess the effect of $H$. pylori eradication among migraine subgroups with and without aura. We used several exclusion criteria that restricted the sample size.

Gasbarrini et al. showed that $H$. pylori eradication therapy could significant reduce the frequency and severity of migraine attacks within a 6-month follow-up [15]. Gabrielli et al. conducted a clinical trial on 148 outpatients with clinical evidences of migraine headache and $H$. pylori infection and reported a significant decrease in severity and frequency of migraine headache after $H$. pylori eradication [16]. Tunca et al. reported a reduction in the symptom of migraine headache in $84.6 \%$ of patients who underwent $H$. pylori eradication and in $75 \%$ of patients who had benefited from treatment [17]. Asghari et al. performed another clinical trial on 108 migraine patients who were infected with $H$. pylori and reported a significant improvement in migraine attacks two months after $H$. pylori eradication [18]. Munno et al. reported that high interleukin (IL)-10 levels were seen in 52.2\% of the patients with migraine headache and showed that treatment with sumatriptan could reduce the level of IL-10. This issue suggested that IL-10 might play a role in the pathogenesis of migraine [19].

Gasbarrini et al. showed that CagA positive H. pylori strains are strongly associated with migraine with aura [9]. This can be explained by a higher inflammatory response of the gastric mucosa to more virulent strains, which release substances that may act as triggers of vasospasm in cerebral arteries, probably implicated in the aura phenomenon. In addition, Hosseinzadeh et al. showed that active $H$. pylori infection is strongly related to outbreak and severity of migraine headache and $H$. pylori treatment can reduce the severity and frequency of migraine headaches significantly [20].

The statistical power was strong enough in both groups [UBT$(\mathrm{P}<0.001)$ and $\mathrm{UBT}+(\mathrm{P}=0.002)]$ particularly in the UBT- subgroup $(n=71)$. However, the sample size was small $(n=9)$ in the UBT+ subgroup. Part of the strong power in this subgroup was due to the matched design of the study and using a paired t-test. Indeed, patients were compared with themselves. This reduced the between groups variance and hence increased the statistical power. Nonetheless, the sample size was not large enough in the UBT+ subgroup and the results of this subgroup should be interpreted with caution.

The main limitation of this clinical trial was the short duration of follow-up. We are not sure whether the reduction in the severity and frequency of headache was permanent or temporary after $H$. pylori eradication. Another limitation of this trial was the before-after design in which the patients were compared with themselves rather than a control group. This may raise the possibility of selection bias. Furthermore, the severity and frequency of headache were evaluated subjectively through history taking. This might introduce information bias into the results. Despite its limitations, this trial could efficiently indicate the effect of $H$. pylori eradication on reduction of migraine attack.

\section{Conclusion}

This trial indicated that $H$. pylori eradication can efficiently reduce the severity and frequency of migraine headache shortly after treatment in patients who are infected with $H$. pylori. Furthermore, the reduction in severity and frequency of headache was much greater in patients with negative UBT compared to those with positive UBT. However, the long-term follow-up studies are required to assess the long term effect of the $H$. pylori eradication on migraine headache.

\section{References}

1. Gasbarrini A, Franceschi F, Gabrielli M (2000) Helicobacter pylori infection and migraine. CNS Drugs 13: 92-101.

2. Tunca A, Turkay C, Tekin O, Kargili A, Erbayrak M (2004) Is Helicobacter pylori infection a risk factor for migraine? A case-control study. Acta Neurol Belg 104: 161-164.

3. Yiannopoulou KG, Efthymiou A, Karydakis K, Arhimandritis A, Bovaretos N, et al. (2007) Helicobacter pylori infection as an environmental risk factor for migraine without aura. J Headache Pain 8: 329-333. 
Citation: Mazaheri S, Majlesi A, Poorolajal J, Hemati N, Khalilian A. Effect of Helicobacter pylori Eradication on Migraine Headache: A Before -After Clinical Trial. J Neurol Psychol. 2014;2(2): 4.

4. Faraji F, Zarinfar N, Zanjani AT, Morteza A (2012) The effect of Helicobacter pylori eradication on migraine: a randomized, double blind, controlled trial. Pain Physician 15: 495-498.

5. Gabrielli M, Fiore G, Candelli M, Giacovazzo M, Pola P, et al. (2002) Re: "Chronic Helicobacter pylori infection and migraine: a case-control study" (Pinessi L, Savi L, Pellicano R, et al. Headache. 2000;40:836-839). Headache 42: $236-237$

6. Gasbarrini A, Franceschi F, Armuzzi A, Ojetti V, Candelli M, et al. (1999) Extradigestive manifestations of Helicobacter pylori gastric infection. Gut 45 I9-I12.

7. Gasbarrini G, Racco S, Franceschi F, Miele L, Cammarota G, et al. (2010) Helicobacter pylori infection: from gastric to systemic disease. Recenti Prog Med 101: 27-33.

8. Pinessi L, Savi L, Pellicano R, Rainero I, Valfrè W, et al. (2000) Chronic Helicobacter pylori infection and migraine: a case-control study. Headache 40: 836-839.

9. Realdi G, Dore MP, Fastame L (1999) Extradigestive manifestation of Helicobacter pylori infection: fact and fiction. Dig Dis Sci 44: 229-236.

10. Suzuki H, Franceschi F, Nishizawa T, Gasbarrini A (2011) Extragastric manifestations of Helicobacter pylori infection. Helicobacter 16: 65-69.

11. Gasbarrini A, Gabrielli M, Fiore G, Candelli M, Bartolozzi F, et al. (2000) Association between Helicobacter pylori cytotoxic type I Cag A positive strains and migraine with aura. Cephalalgia 20: 561-565.

12. Kemper RH, Meijler WJ, Korf J, Ter Horst GJ (2001) Migraine and function of the immune system: A meta-analysis of clinical literature published between 1966 and 1999. Cephalalgia 21: 549-557.

13. Ruggiero P (2010) Helicobacter pylori and inflammation. Curr Pharm Des 16 4225-4236.

14. Prelipcean CC, Mihai C, Gogalniceanu P, Mitrica D, Drug VL, et al. (2007) Extragastric manifestations of Helicobacter pylori infection. Rev Med Chir Soc Med Nat lasi 111: 575-583.

15. Gasbarrini A, De Luca A, Fiore G, Gambrielli M, Franceschi F, et al (1998) Benefificial effects of Helicobacter pylori eradication on migraine. Hepatogastroenterology 45: 765-770.

16. Gabrielle M, Franceschi F, Fior G, Candelli M, Armuzzi A, et al. (2001) Beneficial effects of Helicobacter pylori eradication on migraine: a 12-month follow-up study. J Headache Pain 2: 39-43.

17. Tunca A, Ardicoglu Y, Kargili A, Adam B (2007) Migraine, Helicobacter pylori and oxidative stress. Helicobacter 12: 59-62.

18. Asghari N, Nassaji M, Shojaei H, Mosavi Sh, Ghorbani R (2013) The effect of Helicobacter pylori eradication on migraine without aura. Iran J Neurol 12: 65

19. Munno I, Marinaro M, Bassi A, Cassiano MA, Causarano V, et al. (2001) Immunological aspects in migraine: increase of IL-10 plasma levels during attack. Headache 41: 764-767.

20. Hosseinzadeh M, Khosravi A, Saki K, Ranjbar R (2011) Evaluation of Helicobacter pylori infection in patients with common migraine headache. Arch Med Sci 7: 844-849.

\section{Acknowledgements}

This was part of the MD thesis. We would like to appreciate the Vice-chancellor of Education for technical support and the Vicechancellor of Research and Technology of Hamadan University of Medical Sciences for financial support of this work. We also thank Dr. Torabian and our colleagues who helped us with data collection and analysis.

This study was funded by the Vice-chancellor of Research and Technology, Hamadan University of Medical Sciences. 